\title{
Ensuring rational introduction and responsible use of new TB tools: outcome of an ERS multisector consultation
}

\author{
Giovanni Battista Migliori', Christian Lienhardt², Karin Weyer², \\ Marieke J. van der Werf ${ }^{3}$, Francesco Blasi ${ }^{4}$ and Mario C. Raviglione ${ }^{2}$
}

Affiliations: ${ }^{1}$ WHO Collaborating Centre for TB and lung diseases, Fondazione S. Maugeri, Care and Research Institute, Tradate, Italy. ${ }^{2}$ Global TB Programme, World Health Organization, Geneva, Switzerland. ${ }^{3}$ European Centre for Disease Prevention and Control, Stockholm, Sweden. ${ }^{4}$ Dept of Pathophysiology and Transplantation, University of Milan, IRCCS Fondazione Cà Granda, Milan, Italy.

Correspondence: Giovanni Battista Migliori, WHO Collaborating Centre for Tuberculosis and Lung Diseases, Fondazione Salvatore Maugeri, Care and Research Institute, via Roncaccio 16, 21049, Tradate (VA), Italy.

E-mail: giovannibattista.migliori@fsm.it

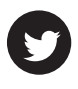

@ERSpublications

ERS multisector consultation to discuss issues relevant to rational introduction and responsible use of new TB tools http://ow.ly/AklCO

In 2014, at the time the World Health Organization (WHO) launched the new global strategy for tuberculosis (TB) prevention, care and control after 2015, 3500 people are still dying from TB every day, despite the remarkable progress in controlling the disease over the past two decades [1-5]. The new WHO strategy promotes the concept of TB elimination ( $<1$ case per million) by 2050, which a group of "low TB incidence countries" (defined as having less than 100 TB cases per million population per year) has the potential to reach ahead of time if they implement a package of interventions aimed at strengthening their current care and control efforts [6-8]. This is in line with the European Centre for Disease Prevention and Control (ECDC) Framework Action Plan to Fight Tuberculosis in the European Union, which was launched in 2008 [9].

Enormous progress has been made in the last decade with a remarkable surge in the development of new diagnostics and medicines [10-12] that can provide the necessary stimulus to optimise TB control and accelerate progress towards elimination. However, as past experience with decades of TB control has shown, improper and irrational introduction and use of these new tools could impair their potential impact and benefit to patients $[1,13-15]$. National authorities, regulators and public health agencies play a key role in ensuring that new tools are implemented properly alongside the existing ones. However, the role and linkage between developers and medical professionals has seldom been considered as an opportunity to further strengthen the implementing environment.

To address this, a symposium was organised by the European Respiratory Society, with the participation of supranational agencies such as WHO and ECDC, to bring together industry and key TB stakeholders to discuss and agree on common principles of rational introduction and responsible use of new TB tools (fig. 1).

The case for rational introduction and use of medicines

Drug resistance in Mycobacterium tuberculosis occurs through spontaneous chromosomal mutations, and depending on the drug target, resistance to a specific drug occurs at a specific rate (i.e. every $x$ number of bacilli will have a mutation-conferring resistance to a specific drug); this spontaneous emergence is reported

Received: July 212014 | Accepted: July 222014

Conflict of interest: None declared.

Copyright @ERS 2014 


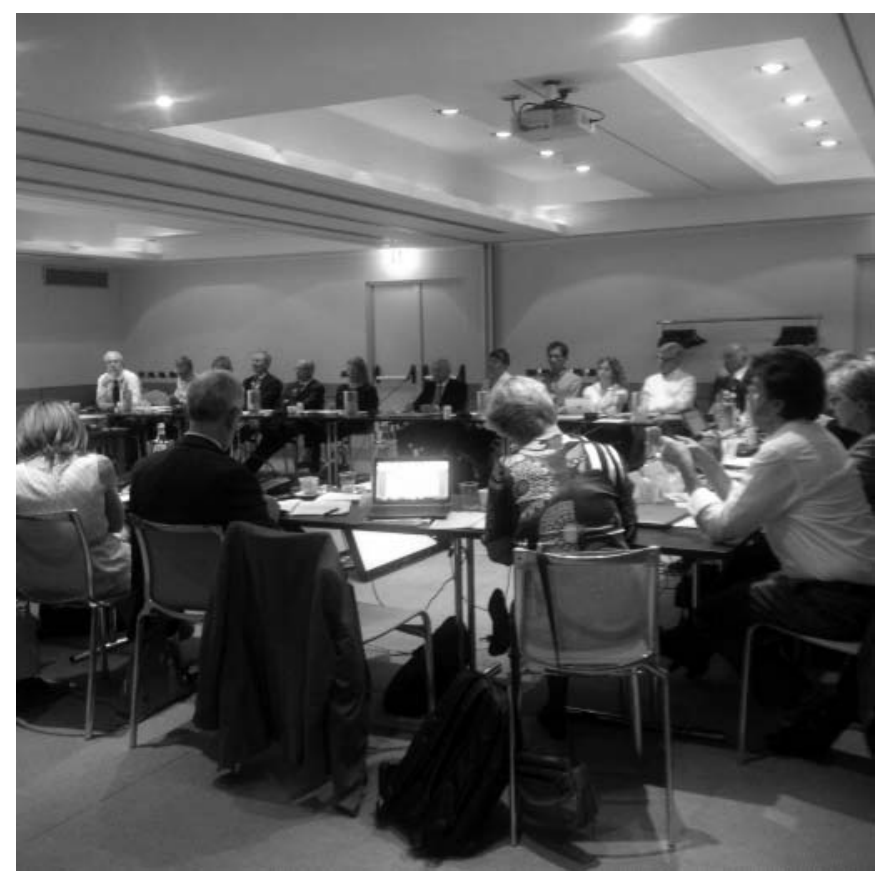

FIGURE 1 Symposium organised by the European Respiratory Society, with the participation of supranational agencies such as the World Health Organization and the European Centre for Disease Prevention and Control, to bring together industry and key tuberculosis (TB) stakeholders to discuss and agree on common principles of rational introduction and responsible use of new TB tools.

to range from one in $10^{6}$ to one in $10^{9}$ cell divisions, depending on the drug [16]. This, in itself, forms the basis and rationale for multidrug TB regimens, targeting the bacilli from several angles to ensure all bacilli are killed, thus preventing the emergence of resistance [16]. Evidence exists that development of multidrugresistant (MDR)-TB can be caused by inadequate treatment, given the drug susceptibility pattern of M. tuberculosis [17-21]. To prevent this, WHO has been regularly publishing guidelines that recommend the best treatment options $[22,23]$.

A recent systematic meta-analysis [17] revealed that the percentage of TB patients receiving an inappropriate treatment regimen is alarmingly high. Prescription of inappropriate treatment regimens was reported in a majority (67\%) of 37 studies from 22 countries covering almost all continents, with the proportion of patients receiving inappropriate regimens ranging between $0.4 \%$ and $100 \%$. Patients who received an inappropriate treatment regimen had a 27 -fold increased risk of developing MDR-TB (risk ratio 26.7, 95\% CI 5.0-141.7) [17].

A recent ECDC systematic review [20] assessed the knowledge of appropriate TB drug regimens (i.e. complying with national or WHO guidelines as per the choice of drugs, doses and treatment duration) among all categories of healthcare workers. Inappropriate knowledge of treatment regimens or of treatment duration was reported in $8-100 \%$ and $5-99 \%$ of healthcare workers, respectively. Knowledge of appropriate doses was also insufficient in most studies analysed in the review [20].

The use of TB drugs for non-TB indications is also contributing to the loss of efficacy of these drugs. A recent review showed that $\mathrm{TB}$ patients exposed to fluoroquinolones for lower respiratory tract infections other than TB had a three-fold higher risk of having fluoroquinolone-resistant TB compared with those not exposed to fluoroquinolones (OR 2.81, 95\% CI 1.47-5.39) [21].

From this, it is clear that new drugs finally appearing on the market run a high risk of losing their efficacy and added value due to development of resistance, if used inappropriately.

In order to address this major public health challenge, WHO has decided to promote actively rational introduction and responsible use of new TB drugs through a coherent series of activities (tables 1 and 2).

First, WHO has been developing recommendations for introduction and use of new drugs or drug regimens that are proposed for the treatment of drug-susceptible or drug-resistant TB, through timely evaluation of novel products and issue of policy guidance on the use of these products [24]. This relies on an open dialogue between the drug/regimen developers and WHO to ensure that sound evidence is available and shared. 
TABLE 1 The World Health Organization's comprehensive six-point policy package to contain and prevent antimicrobial resistance

Commit to a comprehensive, financed national plan with accountability and civil society engagement

Strengthen surveillance and laboratory capacity

Ensure uninterrupted access to quality essential medicine

Regulate and promote rational use of medicines, including those used in animal husbandry, and ensure proper patient care

Enhance infection prevention and control

Foster innovations, and the research and development of new tools

Reproduced from [13] with permission from the publisher.

Second, the issue of policy recommendation is followed by: 1) the development of operational guidance for the roll-out of new TB drugs or regimens in countries ensuring rational introduction and responsible use; and 2) support to piloted deployment of new drugs/regimens in diverse settings. This work is carried out in close collaboration with key stakeholders at a national level, including TB control programmes, drug regulatory authorities and representatives of professional organisations, to promote rapid access to new therapies that are safe and efficacious while preventing emergence of new resistance.

Third, these activities take place under an overarching WHO policy implementation package that was developed to support national efforts towards the implementation of recommended drugs and/or regimens. The package aims to complement existing and new policy guidance on the use of new drugs for the treatment of $\mathrm{TB}$, and provides the key elements for introduction of new TB drugs and/or regimens for wide use in countries. It consists in a set of six elements required for optimal and rational introduction of new TB drugs.

Based on this approach, in June 2013, WHO issued interim guidance on the use of bedaquiline for the treatment of patients with MDR-TB that includes recommendations for responsible use, and work has started with a series of "early implementing countries" committed to introduce the drug according to such recommendations [15]. Work is currently underway to develop interim guidance on delamanid.

Similarly, to support European Union and European Economic Area Member States in the process of introducing new drugs into their TB control programmes and/or strategies, ECDC is developing guidance

TABLE 2 The World Health Organization (WHO) policy implementation package for rational introduction of new tuberculosis (TB) drugs

\section{Minimum requirements for country preparedness and planning}

\section{Development of an implementation plan for introduction of new TB drugs or regimens}

\section{Monitoring and evaluation of new drugs and regimens, including pharmacovigilance and drug resistance surveillance}

Private sector engagement

\author{
Systems approach for ensuring uninterrupted supply of new \\ and existing quality-assured medicines \\ Operational research
}

A set of basic health and programmatic capacities must be in place at the country level for the optimal introduction and implementation of a new TB drug or drug regimen according to WHO policy recommendations

This national implementation plan must take into account the various operational models for introduction of new TB drugs or regimens, depending on the TB epidemics situation and level of preparedness in a country, and the type of drug or regimen of drugs to be introduced

Introduction of new TB drugs or regimens requires careful monitoring of safety (particularly if drugs are being introduced following phase Ilb trials), based on active pharmacovigilance, and of drug resistance.

Introduction of new TB drugs requires a set of best practice regulations from the local ministry of health to provide rational access and

protection of new drugs, particularly in the context of substantial involvement of the private sector in TB care

This describes the need for a clearly established procurement and supply chain management system at country level

Can assist in the implementation and scale-up processes and help support evaluation of public health impact, through the collection of relevant information to measure feasibility, cost-effectiveness, acceptability and impact of the new drugs or regimens 
on options for this. This guidance will reflect the rationale and importance of carefully planned introduction of new drugs, as well as providing expert opinions on the options for optimal introduction of new drugs/regimens in TB control programmes.

From this, it is clear that drug resistance can be controlled by widely adopting the highest standards of care [25-27], issuing bold health policies and, where necessary, strict and enforced regulations for the introduction and use of new and repurposed TB drugs (e.g. fluoroquinolones, linezolid, meropenem, etc.) $[21,28-31]$.

\section{The case for ethical and correct use of diagnostics}

Appropriate treatment of patients and effective introduction of new drugs and regimens are premised on accurate and rapid diagnosis. This is particularly important for drug-resistant tuberculosis, where incorrect diagnosis or drug susceptibility testing (DST) results have dire consequences.

Contrary to the drugs pipeline, TB diagnostic test development has seen a proliferation of new technologies over the past 5 years, and currently more than 50 new TB tests are in various stages of development. In 2008, WHO established a structured process to rapidly review evidence from laboratory and field trials of new diagnostics to ensure that accurate, reproducible tests are introduced effectively, while also strongly advising against tests found to be suboptimal [32].

Molecular diagnostics for TB and DST hold great promise, especially given their speed and potential robustness for use in decentralised settings. The Xpert MTB/RIF assay (Cepheid, Sunnyvale, CA, USA), endorsed by WHO in late 2010, has been a game-changer for test development, illustrating the benefit of close collaboration between academia, industry and end-users [33]. Nevertheless, currently available TB tests are not mutually exclusive and one size no longer fits all. Rational introduction of new tests requires assessment of appropriate diagnostic algorithms to ensure optimal patient access and the use of different tests at different levels of the laboratory service [32].

In addition, rational introduction of new $\mathrm{TB}$ diagnostics requires targeted testing, effective referral mechanisms for additional testing and rapid communication of results to healthcare providers. These requirements are becoming increasingly important in view of anticipated new regimens, where rapid diagnosis (and exclusion of drug resistance) will be imperative to protect both patients and the efficacy of new drugs. Point-of-care DST technologies with clearly defined target product profiles (TPPs) are essential to guide the choice of drugs and regimens to be used in the future. The outcomes of an international consensus document on TPPs for new TB tests, currently under preparation following a meeting convened by WHO in April 2014, are eagerly awaited, as is the development of standardised DST for new drugs already approved by regulatory authorities.

Laboratory capacity strengthening should go hand-in-hand with introduction of new drugs and regimens. The EXPAND-TB Project, a multi-donor, multi-country laboratory strengthening project managed by WHO has clearly shown that sophisticated reference laboratory infrastructure can be effectively established in developing countries [34] and that contemporary technologies, such as liquid culture and line probe assays, can be effectively transferred when multiple stakeholders join the same effort.

At the European Union (EU) level, new diagnostics are quickly evaluated for their applicability in the EUspecific setting by the European Reference Laboratory Network for TB. Thereafter, they are included in the handbook Mastering the Basics of TB Control [35].

Lastly, the holy grail of new TB test development, an accurate, robust and safe point-of-care test to monitor treatment response, especially in patients with drug-resistant disease, remains elusive. All currently available new TB diagnostics fail in this regard, requiring elaborate and sophisticated laboratory capacity for conventional culture and DST to be established in conjunction with the roll-out of new diagnostic tests. A "diagnosis to cure" strategy aligning diagnostic and drug development would therefore be imperative to meet the research and innovation objectives of the new post-2015 WHO global strategy and achieve the ultimate target of TB elimination.

\section{Seven principles for future action}

From the above, it is clear that the introduction of new TB tools in a responsible way requires that efforts be made by all concerned parties so that the efficacy and effectiveness of TB treatment with optimised drug regimens is aligned with the use of simplified platforms for diagnosis, and adapted delivery systems. This requires early engagement of all stakeholders in the preparation of policies for the introduction of new TB tools. These policies must be based on sound evidence and promote measures for optimal delivery, including quality assurance and procurement. National authorities then have a key role to play in ensuring that introduction of new tools follows policy recommendations and that appropriate plans are made. 
A common statement of principles was agreed upon to guide future interaction between industry engaged in research and development and global TB stakeholders in ensuring that the impact of new tools is maximised through their rational and ethical use.

Recognising the challenges faced, participants of the symposium agreed to a seven-point agenda for consideration and future action:

1) commitment to ethical and responsible use of new and existing diagnostics;

2) commitment to support and provide the best conditions for rational introduction and responsible use of new and existing TB drugs and regimens;

3) alignment of market objectives of industry with public health needs;

4) early collaboration between diagnostic and drug developers to maximise synergy (for instance, in guaranteeing development of DST simultaneously with that of the new agent);

5) early interaction of WHO and partner agencies with industry for definition of TPPs for new diagnostics and drugs, as well as proper strategies for introduction;

6) reinforcement of the role of WHO and professional organisations in ensuring adherence of healthcare providers to international standards of diagnosis, treatment and care; and

7) establishment of a forum with regular meetings bringing together respiratory professionals, industry and global TB stakeholders to address issues relevant to rational introduction and responsible use of new TB tools nationally and globally.

A promising new era in TB diagnostic and drug development has begun. It is now critical to consolidate recent progress and ensure that new tools for $\mathrm{TB}$ diagnosis and treatment are suitably introduced in individual countries in a way that guarantees access to the best care for all those in need and avoids inappropriate use. Programmatic implementation of new TB tools should be aligned with ongoing efforts that aim to maximise the efficiency and effectiveness of tuberculosis care by advancing point-of-care and other simplified platforms for diagnosis and monitoring, optimising drug regimens, reducing costs, adapting delivery systems and mobilising communities. This will not be possible without clear and strong commitment of all stakeholders to ensure that evidence-based strategies for introduction of new tools are linked to wide access and responsible use.

\section{Acknowledgements}

The authors wish to thank Lia D'Ambrosio and Rosella Centis (WHO Collaborating centre for Tuberculosis and Lung Diseases, Fondazone S. Maugeri, Care and Research Institute, Tradate, Italy), and Chris Gilpin, Fuad Mirzayev and Wayne Van Gemert (Global TB Programme, World Health Organization, Geneva, Switzerland) for their useful comments on the manuscript.

\section{References}

1 World Health Organization. Strategic and Technical Advisory Group for TB meets in Geneva. www.who.int/tb/ features_archive/stag_2014/en Date last accessed: July 2, 2014. Date last updated: June 23, 2014.

2 Sotgiu G, Woodhead M. Good news about a bad subject: scientific evidence to help defeat multidrug/extensively drug-resistant tuberculosis. Eur Respir J 2014; 44: 5-7.

3 Tanimura T, Jaramillo E, Weil D, et al. Financial burden for tuberculosis patients in low- and middle-income countries: a systematic review. Eur Respir J 2014; 43: 1763-1775.

4 World Health Organization. Global Tuberculosis Report 2013. Document WHO/HTM/TB/2013.11. Geneva, WHO, 2013.

5 World Health Organization. Global strategy and targets for tuberculosis prevention, care and control after 2015. http://apps.who.int/gb/ebwha/pdf_files/EB134/B134_12-en.pdf?ua=1 Date last accessed: July 2, 2014. Date last updated: November 29, 2013.

6 Diel R, Loddenkemper R, Zellweger JP, et al. Old ideas to innovate tuberculosis control: preventive treatment to achieve elimination. Eur Respir J 2013; 42: 785-801.

7 D’Ambrosio L, Dara M, Tadolini M, et al. Tuberculosis elimination: theory and practice in Europe. Eur Respir J 2014; 43: 1410-1420.

8 Voniatis C, Migliori GB, Voniatis M, et al. Tuberculosis elimination: dream or reality? The case of Cyprus. Eur Respir J 2014; 44: 543-546.

9 European Centre for Disease Prevention and Control. Framework action plan to fight tuberculosis in the European, Union. Stockholm, ECDC, 2008.

10 Weyer K, Mirzayev F, Migliori GB, et al. Rapid molecular TB diagnosis: evidence, policy making and global implementation of Xpert MTB/RIF. Eur Respir J 2013; 42: 252-271.

11 UNITAID. Tuberculosis Diagnostic Technology and Market Landscape: Semi-annual update. 2013. www.unitaid. eu/images/marketdynamics/publications/UNITAID-TB_Dx_Landscape-Update_Dec\%202013.pdf Date last accessed: July 2, 2014. Date last updated: December 2013.

12 UNITAID. Tuberculosis Medicines Technology and Market Landscape: 1st edition. 2013. www.unitaid.eu/images/ marketdynamics/publications/UNITAID-TB_Medicines_Landscape-1st_edition.pdf Date last accessed: July 2, 2014. Date last accessed: September 2013. 
13 Raviglione MC, Lange C, Migliori GB. Preventing and managing antimicrobial resistance: imperative for chest physicians. Eur Respir J 2011; 37: 978-981.

14 Leung E, Weil DE, Raviglione M, et al. The WHO policy package to combat antimicrobial resistance. Bull World Health Organ 2011; 89: 390-392.

15 World Health Organization. The use of bedaquiline in the treatment of multidrug-resistant tuberculosis: interim policy guidance. Document WHO/HTM/TB/2013.6. Geneva, WHO, 2013.

16 Colijn C, Cohen T, Ganesh A, et al. Spontaneous emergence of multiple drug resistance in tuberculosis before and during therapy. PLoS ONE 2011; 6: e18327.

17 Van der Werf MJ, Langendam MW, Huitric E, et al. Multidrug resistance after inappropriate tuberculosis treatment: a meta-analysis. Eur Respir J 2012; 39: 1511-1519.

18 Matthys F, Rigouts L, Sizaire V, et al. Outcomes after chemotherapy with WHO category II regimen in a population with high prevalence of drug resistant tuberculosis. PLoS One 2009; 4: e7954.

19 Cox HS, Niemann S, Ismailov G, et al. Risk of acquired drug resistance during short-course directly observed treatment of tuberculosis in an area with high levels of drug resistance. Clin Infect Dis 2007; 44: 1421-1427.

20 Langendam MW, van der Werf MJ, Huitric E, et al. Prevalence of inappropriate tuberculosis treatment regimens: a systematic review. Eur Respir J 2012; 39: 1012-1020.

21 Migliori GB, Langendam MW, D’Ambrosio L, et al. Protecting the tuberculosis drug pipeline: stating the case for the rational use of fluoroquinolones. Eur Respir J 2012; 40: 814-822.

22 World Health Organization. Treatment of tuberculosis: guidelines for national programmes. Fourth Edition. Document WHO/HTM/TB/2009.420. Geneva, WHO, 2010.

23 Falzon D, Jaramillo E, Schünemann HJ, et al. WHO guidelines for the programmatic management of drug-resistant tuberculosis: 2011 update. Eur Respir J 2011; 38: 516-528.

24 World Health Organization. Introduction and rational use of new drugs/regimens for the treatment of tuberculosis in countries. www.who.int/tb/new_drugs/en/ Date last accessed: July 2, 2014.

25 TB CARE I. International Standards for Tuberculosis Care, Edition 3. The Hague, TB CARE I, 2014.

26 Migliori GB, Zellweger JP, Abubakar I, et al. European union standards for tuberculosis care. Eur Respir J 2012; 39: $807-819$.

27 Van der Werf MJ, Sandgren A, D’Ambrosio L, et al. The European Union standards for tuberculosis care: do they need an update? Eur Respir J 2014; 43: 933-942.

28 Sotgiu G, Centis R, D'Ambrosio L, et al. Efficacy, safety and tolerability of linezolid containing regimens in treating MDR-TB and XDR-TB: systematic review and meta-analysis. Eur Respir J 2012; 40: 1430-1442.

29 Lee M, Lee J, Carroll MW, et al. Linezolid for treatment of chronic extensively drug-resistant tuberculosis. N Engl J Med 2012; 367: 1508-1518.

30 Tang S, Yao L, Hao X, et al. Efficacy, safety and tolerability of linezolid for the treatment of XDR-TB: a study in China. Eur Respir J 2014 [In press DOI: 10.1183/09031936.00035114].

31 De Lorenzo S, Alffenaar JW, Sotgiu G, et al. Efficacy and safety of meropenem-clavulanate added to linezolidcontaining regimens in the treatment of MDR-/XDR-TB. Eur Respir J 2013; 41: 1386-1392.

32 World Health Organization. Policy Framework for Implementing New Tuberculosis Diagnostics: March 2010 www.who.int/tb/laboratory/whopolicyframework_rev_june2011.pdf Date last accessed: July 2, 2014.

33 Weyer K, Carai S, Nunn P. Viewpoint TB diagnostics: what does the world really need? J Infect Dis 2011; 204: Suppl. 4, S1196-S1202.

34 World Health Organization. The EXPAND-TB Project Progress and Impact Brief. 2014. www.who.int/campaigns/ tb-day/2014/tb-brochure.pdf Date last accessed: July 2, 2014.

35 European Centre for Disease Prevention and Control. Masterin the Basics of TB Control. www.ecdc.europa.eu/en/ publications/Publications/1105_TER_Basics_TB_control.pdf 